\title{
Role of Diffusion Weighted Magnetic Resonance Imaging in Evaluation of Bone Marrow Edema of the Knee
}

Fathy Hussein Ali ${ }^{1}$, Abdullah Hussein Ahmed ${ }^{1}$, Khaled Aly Matarawy ${ }^{2}$, Walid Mohamed Ahmed ${ }^{2}$, Marwa Fadl Elsayed Hashad ${ }^{1 *}$

${ }^{1}$ Department of Radiodiagnosis - Faculty of Medicine, Azhar University for Girls

${ }^{2}$ Department of Radiodiagnosis - Medical Research Institute, Alexandria University

*Corresponding author: Marwa Fadl Elsayed Hashad, Mobile: (+20) 01005216383, E-Mail: marwafadlhashad@gmail.com

\begin{abstract}
Background: Bone marrow edema (BME) has not been recognized as a significant pain generator or as a primary contributor to disease until recently. Bone marrow edema is characterized by a non-specific pattern of ill-defined high signal intensity on short-tau inversion-recovery and T2-weighted magnetic resonance (MR) images and low signal intensity on T1-weighted images.

Objective: To assess the value of diffusion weighted magnetic resonance imaging in the diagnosis and evaluation of bone marrow edema at the knee region.

Patients and methods: This study was conducted on 130 patients referred with clinical suspicion of knee edema with or without history of trauma. They were divided into group "A" with lesions less than $15 \mathrm{~mm}$ and group "B" with lesions greater than $15 \mathrm{~mm}$. Their ages ranged between 9 years and 75 years with mean age of 42 years old. They were 43 females and 87 males.

Results: Between both groups A and B, we found that bone marrow lesions (BMLs) were approximately two times larger on apparent diffusion coefficient (ADC) maps than on FS PD-weighted TSE images. The most important value of adding the ADC-derived maps in routine MRI examination of the knee is to show additional bone marrow lesions that only can be detected at the ADC maps and could not be detected at the FS-PD. About 27 more lesions in 27 patients and one patient showed two more lesions. These non-concordant lesions accounted totally for 29 more bone marrow lesions.

Conclusion: Magnetic resonance imaging (MRI) is the mainstay of diagnosis, staging and management plan of knee traumatic and non-traumatic disorders. ADC maps are more sensitive than corresponding FS PD weighted TSE images allowing for detection of significantly more and larger BMLs. In addition, they represent a valuable add-on in knee imaging protocols.
\end{abstract}

Keywords: Bone marrow edema, Knee, Diffusion weighted magnetic resonance imaging, ADC, FS PD-weighted.

\section{INTRODUCTION}

Bone marrow edema (BME) is emerging as an important clinical issue because of its contribution to patient symptoms and its capacity to accelerate the progression of certain diseases. Differential diagnosis through appropriate imaging is vital for case management and may contribute in the prevention or decreased progression of certain pathologies. It is important to understand the breadth of diseases exhibiting BME, the risk factors for these diseases and the role of imaging in differential diagnosis ${ }^{(1)}$. When located adjacent to a joint, BME is often associated with pain or discomfort. While the appearance of associated pathologies can vary and the appearance of BME itself is consistent ${ }^{(2)}$.

MRI is ideal for localizing the increased extracellular water of BME and for the differential diagnosis of the associated disease ${ }^{(3)}$.

BME possesses an intermediate signal (higher than muscle or disc) on T1-weighted imaging and a high signal on T2-weighted imaging. On fatsuppressed (FS) imaging, contrast-enhanced imaging, and short-tau inversion recovery (STIR), BME appears hyperintense when compared to normal marrow. STIR has been advised due to its lower cost and lack of risk to the patient for a reaction to Gadolinium ${ }^{(4)}$.

MRI findings often cannot determine the precise underlying condition. Therefore, correlation with clinical history, biochemical markers, and other imaging modalities are required. Understanding of the patterns of marrow abnormalities in the knee can help narrow the list of differential diagnoses and guide the radiologist toward an appropriate treatment recommendation ${ }^{(5)}$.

Unlike standard MR imaging sequences, in diffusion-weighted imaging (DWI), the signal depends on the random movement of water molecules and tissue susceptibility. These functional diffusion data can be displayed quantitatively on apparent diffusion coefficient (ADC) maps. Intact bone marrow shows directional diffusion, whereas BML shows isotropic diffusion, which suggests that the osseous trabeculae impose a physiological diffusion barrier. While, trabecular injury facilitates enhanced isotropic 
diffusion whatever the cause of injury including ischemic, mechanical and reactive cause ${ }^{\left({ }^{6}\right)}$. The diagnostic benefit of ADC map evaluation in comparison to fat- saturated (FS) PD weighted TSE image has not been well adequately established in the previous literature. And because of short acquisition times, ADC maps might serve as add-ons to FS PDweighted TSE sequences in knee imaging protocols through improving detection of small BMLs and potentially allowing assessment of areas impaired by artifacts or in homogeneous fat saturation ${ }^{(5)}$.

The aim of this study was to assess the value of diffusion weighted magnetic resonance imaging in the diagnosis and evaluation of bone marrow edema at the knee region.

\section{PATIENTS AND METHODS}

This study was conducted on 130 patients presented to private orthopedic clinics with clinical suspicion of bone marrow edema and referred to private Radiology Centers (Mabarret El-Asafra and Al-Madina Scan Centers) for MRI evaluation in the time interval from July 2017 to May 2019.

Our study included 130 patients referred with clinical suspicion of knee edema with or without history of trauma. Their ages ranged between 9 years and 75 years; with mean age 42 . They were 43 females and 87 males. We divided the patients into two groups, group " $\mathrm{A}$ " with lesions less than $15 \mathrm{~mm}$ that included 46 patients, and group "B" that showed BMLs more than $15 \mathrm{~mm}$ and included 74 patients.

13 patients out of the 130 were subjected to MRI knee study using an Espree $1.5 \mathrm{~T}$ closed magnet MRI scanner (Siemens, Germany) and 2 patients were subjected to Achieva $1.5 \mathrm{~T}$ closed magnet MRI machine (Phillips, Netherlands). The remaining 115 patients were subjected to Smepra $1.5 \mathrm{~T}$ closed magnet (Siemens, Germany). The dedicated knee coil was used as a receiver coil supplied by the manufacturer.

\section{Inclusion criterion}

Patients that were suspected, in routine slandered examination, or diagnosed to have bone marrow edema.

\section{Exclusion criteria}

Post-operative cases. Patients with neoplastic tumors. Any electrically, magnetically or mechanically activated metallic devices such as cardiac pace makers, insulin pump, cochlear implants and neurostimulator. Intra-cranial (non-titanium) aneurysmal clips. Pregnancy (risk versus benefit should be assessed). Ferromagnetic foreign bodies, surgical clips, staples, plates and screws near the field of view. Clostrophobic patients and patients that cannot be immobilized for long times (may be anesthetized). The contraindications to contrast material should be considered as chronic renal failure patients with GFR $>40 \mathrm{ml} / \mathrm{min}$.

All the studied patients were subjected to the following:

1. Adequate history taking.

2. Thorough clinical examination.

3. Conventional MRI examination of the knee.

4. Diffusion-weighted MRI with ADC map.

5. Complementary $\mathrm{X}$ ray or CT was done when needed to assess bony fractures and bony lesions.

Ethical approval and written informed consent: An approval of the study was obtained from Al- Azhar University Academic and Ethical Committee. Every patient signed an informed written consent for acceptance of the operation.

\section{Technique of MRI:}

Patients were scanned in the supine position using a knee coil as the receiver coil and the following protocols were applied:

\section{Each examination included:}

i- Conventional SE MRI sequences include:

- Sagittal T1 WI, PD and Gradient sequences.

- Coronal STIR sequences.

- Axial T2 and PD-FS sequences.

Table (1): The following parameters were utilized in conventional MRI in our study

\begin{tabular}{|c|c|c|c|c|}
\hline & T1 WI & T2 WI & PD FS WI & T2 gradient \\
\hline TR in ms & $448-544$ & $3370-5000$ & $2720-3400$ & 45 \\
\hline TE in ms & $12-17$ & $75-119$ & $26-43$ & 25 \\
\hline Slice thick. & $3-3.5 \mathrm{~mm}$ & $3.5 \mathrm{~mm}$ & $3-3.8 \mathrm{~mm}$ & $3 \mathrm{~mm}$ \\
\hline Flip angle & $90-170^{\circ}$ & $150^{\circ}$ & $90-150^{\circ}$ & $\mathrm{N}$ \\
\hline Gap in mm & $4.3-4.9$ & $4.2-5.4$ & $4.2-4.3$ & $3.3-4.8$ \\
\hline Matrix resol. & $320 \times 216$ & $320 \times 246$ & $320 \times 205$ & $384 \times 326$ \\
\hline FOV & $256 \times 170$ & $384 \times 244$ & $256 \times 256$ & \\
\hline
\end{tabular}




\section{ii- Diffusion weighted MRI:}

Axial and Sagittal DWI with different $b$ values ranging from $b 0, b 50, b 400, b 600, b 800$ and $b 1000$ to obtain best image as well as ADC maps.

A. The diffusion-weighted images were obtained in the sagittal and axial planes by using a single-shot echoplanar sequence with the sensitivity encoding technique. The $b$ values corresponding to the diffusionsensitizing gradient were $0,50,400,600,800$ and 1000 $\mathrm{sec} / \mathrm{mm}^{2}$.

B. Single shot echo planner diffusion weighted imagesderived apparent diffusion coefficient (ADC) mapping for the whole patients.

C. ADC values were measured in $\mathrm{n} \times 10^{-3} \mathrm{~mm}^{2} / \mathrm{s}$. using Horos workstation.

D. Complementary non-contrast CT was used when needed using 64-slice MDCT Siemens.

Table (2): The following parameters were ere utilized in diffusion-weighted MRI used in our study

\begin{tabular}{|c|c|}
\hline TR in ms & $2600-2800$ \\
\hline TE in ms & $80-160$ \\
\hline Slice thickness & $4-5.6 \mathrm{~mm}$ \\
\hline Flip angle & $90^{\circ}$ \\
\hline Matrix resol. & $176 \times 176$ \\
& $188 \times 188$ \\
\hline FOV & 260 \\
\hline Gap & $4-6$ \\
\hline
\end{tabular}

E. The statistical data were processed and are demographically organized, tabulated and statistically analyzed using percentage of total, mean and range for patients' ages, sex, clinical presentations and radiological findings.

Only FS PD-weighted TSE images and ADC maps were used to detect BMLs. T1-weighted images primarily served as an anatomic correlate for ADC map interpretation. Additionally, they allowed identification of other causes of bone marrow edema like degenerative changes, osteonecrosis or subchondral cysts etc., which cannot be discerned from traumatic BMLs on ADC maps alone. If BMLs were located in regions with extensive degenerative changes or any other pathologies than trauma, these lesions were fully analyzed in other conventional MRI sequences.

\section{Statistical analysis}

Data were fed to the computer and analyzed using IBM SPSS software package version 20.0. Qualitative data were described using number and percent. Quantitative data were described using range (minimum and maximum), mean, standard deviation and median. Significance of the obtained results was judged at 5\% level.

\section{RESULTS}

Age and sex distribution:

The study included one patient 9 years old, 10 patients between 10 and 20 years of age, 24 patients between 20 and 30 years of age, 25 patients between 30 and 40 years of age, 23 patients between 40 and 50 years of age, 22 patients between 50 and 60 years of age, 19 patients between 60 and 70 years of age and 6 patients above 70 years age group. The mean age was 42 years. The body weight of our patients ranged from 35 up to $130 \mathrm{~kg}$ with a mean of about $89.4 \mathrm{~kg}$ [Table 3].

Table (3): Distribution of studied patient's according to demographic data

\begin{tabular}{|c|c|c|c|c|c|}
\hline Age & Males & Percent & Females & Percent & Total \\
\hline$<\mathbf{1 0}$ & 1 & $0.8 \%$ & 0 & 0 & 1 \\
\hline $\mathbf{1 0}-$ & 8 & $6.2 \%$ & 2 & $1.5 \%$ & 10 \\
\hline $\mathbf{2 0}-$ & 22 & $16.9 \%$ & 2 & $1.5 \%$ & 24 \\
\hline $\mathbf{3 0}-$ & 21 & $16.2 \%$ & 4 & $3 \%$ & 25 \\
\hline $\mathbf{4 0}-$ & 16 & $12.3 \%$ & 7 & $5.4 \%$ & 23 \\
\hline $\mathbf{5 0}-$ & 7 & $5.4 \%$ & 15 & $11.5 \%$ & 22 \\
\hline $\mathbf{6 0}-$ & 11 & $8.5 \%$ & 8 & $6.2 \%$ & 19 \\
\hline$>\mathbf{7 0}$ & 1 & $0.8 \%$ & 5 & $3.8 \%$ & 6 \\
\hline
\end{tabular}

\section{According to the underlying pathology:}

The patients were mainly categorized according to cause of the bone marrow edema into three distinct pathological groups: 1) Ischemic BME, 2) Mechanical BME and 3) Reactive BME (Table 4).

Table (4): Number of patients according to the type of pathology

\begin{tabular}{|l|c|l|}
\hline Type of pathology & $\begin{array}{c}\text { Number of } \\
\text { Patients }\end{array}$ & Percent \\
\hline Ischemic BME & 49 & $37.7 \%$ \\
\hline Mechanical BME & 32 & $24.6 \%$ \\
\hline Reactive BME & 61 & $46.9 \%$ \\
\hline
\end{tabular}

N.B.: More than one pathology was seen together in the same knee.

\section{According to conventional MRI Findings: \\ Anatomical distribution of the knee edema:}

Radiologically, 116 patients (89.2\%) with knee edema, the affected bone was the femur, 93 of them $(71.5 \%)$ was the tibia, 60 of them $(46.2 \%)$ showed patellar affection and only 13 patients $(10 \%)$ showed fibular affection. Considering more than one bone may be affected in the same patient (Table 5).

Table (5): Distribution of the lesions in knee bones

\begin{tabular}{|c|c|c|}
\hline \multirow{2}{*}{ Anatomical sites } & \multicolumn{2}{|c|}{ No. Of Patients } \\
\cline { 2 - 3 } & No. & $\%$ \\
\hline Patella & 60 & $46.2 \%$ \\
\hline Femur & 116 & $89.2 \%$ \\
\hline Tibia & 93 & $71.5 \%$ \\
\hline Fibula & 13 & $10 \%$ \\
\hline
\end{tabular}

N.B.: More than one bone was affected at the same patient. 


\section{Associated findings with the knee bone marrow edema:}

The examined patients showed other pathologies associated with the bone marrow edema involving the meniscus, ligaments, bone fracture and other findings. About 17 patients (13.1\%) showed only bone marrow edema with no associated findings or any other injuries. Considering more than one pathology involved in the same patient with the knee edema are shown in Table (6).

Table (6): Types of different associated pathologies with the knee bone marrow edema

\begin{tabular}{|c|c|c|}
\hline \multirow{2}{*}{ Associated Pathology } & \multicolumn{2}{|c|}{ No. Of Patients } \\
\cline { 2 - 3 } & No. & $\%$ \\
\hline Meniscal pathology & 101 & $77.7 \%$ \\
\hline Ligamental pathology & 51 & $39.2 \%$ \\
\hline Associated fractures & 11 & $8.4 \%$ \\
\hline Other findings & 9 & $6.9 \%$ \\
\hline Nothing & 17 & $13.1 \%$ \\
\hline
\end{tabular}

N.B.: More than one pathology was seen at the same patient.

\section{According to the DW findings:}

Values: In our study, we tried multiple B-values to figure out the best values for the best evaluation of the bone marrow edema at the DWI. B0, 1000 was tried in 12 patients $(9.2 \%), \mathrm{B} 0,800$ in 13 patients (10\%), B50, $600, \mathrm{~B} 50,400$ and 800 in three patients $(2.3 \%)$ and B50 and 100 in one patient $(0.8 \%)$. While, the most used value with the best appearance in DWI was B50, 800 in 98 patients accounting for $75.4 \%$ of the examined patients (Table 7).

\begin{tabular}{|l|c|c|}
\hline $\begin{array}{c}\text { Table (7): Different } \\
\text { B-values in DWI } \\
\text { B- Values }\end{array}$ & $\begin{array}{c}\text { No of } \\
\text { patients }\end{array}$ & Percent \\
\hline B 0, 1000 & 12 & $9.2 \%$ \\
\hline B 0, 800 & 13 & $10 \%$ \\
\hline B 50, 600 & 3 & $2.3 \%$ \\
\hline B 50, 800 & 98 & $75.4 \%$ \\
\hline B 50, 1000 & 1 & $0.8 \%$ \\
\hline B 50, 400, 800 & 3 & $2.3 \%$ \\
\hline Total & $\mathbf{1 3 0}$ & $\mathbf{1 0 0} \%$ \\
\hline
\end{tabular}

\section{BME appearance pattern in the ADC map:}

Types of bone marrow lesions: In our study, we defined three types of BMLs according to their visual appearance.

- Type I or reticular BMLs were speckled and diffuse areas of signal intensity increase on ADC maps.

- Type II or intermediate BMLs were confluent patches of signal intensity increase.

- Type III or compact BMLs were areas of focal and homogeneous signal intensity increase (possibly accompanied by peripheral, reticular type signal intensity increase) [Figure 1]. ADC values of all major BMLs were obtained by measuring ADC values of the most prominent regions of interest on the ADC maps calculated at a noise level of 0 . We sorted the results according to BML appearance. N.B: Some of the examined patients showed more than one pattern at the same bone affected and in different bones affected at the same patient.

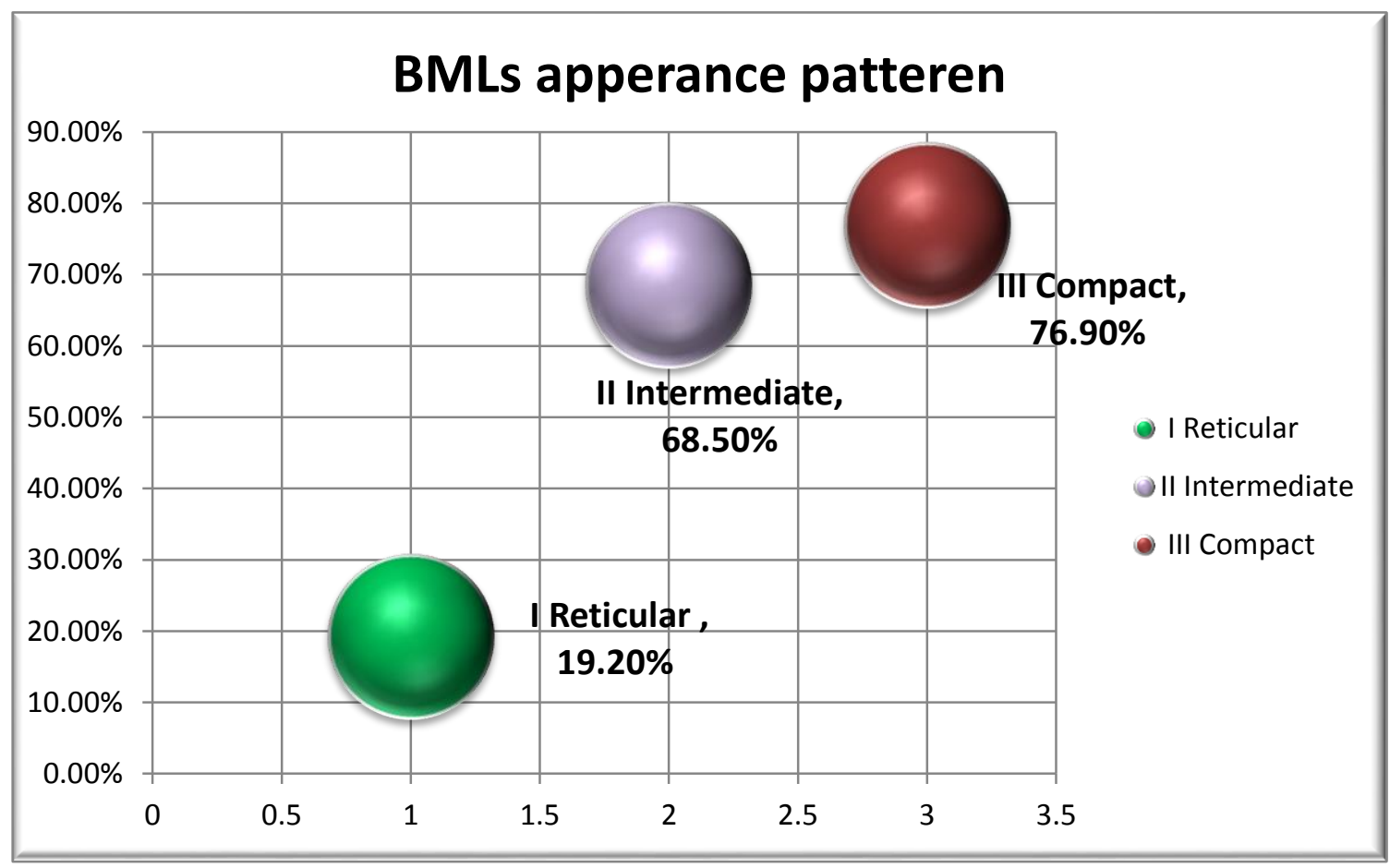

Figure (1): BMLs appearance pattern in ADC maps 


\section{Groups of patients:}

Among the examined 130 patients that showed bone marrow edema detected on the FS-PD sequences, underwent $\mathrm{ADC}$ examination, 10 patients accounting for about $7.7 \%$ of the patients were excluded as follow: nine patients due to technical error within the ADC diffusion sequences and one patient showed restricted signal due to infective osteomyelitis. The remaining 120 patients showed multiple BMLs in the same patient. We selected the largest lesion among the multiple BMLs within the same patient and underwent the analysis below. When analyzing the patients we divided the remaining 120 cases to Group $\boldsymbol{A}$ that showed bone marrow edema at the PD sequences up to $15 \mathrm{~mm}$ at its maximum AP diameter and Group B more than $15 \mathrm{~mm}$ at its maximum AP diameter and underwent further volumetric assessment. Considering the largest lesion in every patient that sometimes showed more than one lesion (Table 8).

Table (8): Number of cases between both study groups

\begin{tabular}{|c|c|c|c|c|c|c|}
\hline \multicolumn{6}{|c|}{ Groups } & Total \\
\hline \multicolumn{2}{|c|}{$\begin{array}{c}<15 \mathrm{~mm} \\
\mathrm{~A}\end{array}$} & \multicolumn{2}{|c|}{$\begin{array}{c}>15 \mathrm{~mm} \\
\text { B }\end{array}$} & \multicolumn{2}{|c|}{ Excluded } & \multirow{2}{*}{130} \\
\hline Count & $\%$ & Count & $\%$ & Count & $\%$ & \\
\hline 46 & $35 \%$ & 74 & $57 \%$ & 10 & $8 \%$ & $100 \%$ \\
\hline
\end{tabular}

\section{ADC values:}

The ADC value of intact bone marrow was measured in 120 patients with exclusion of the previously mentioned ten cases. The mean ADC in intact bone marrow was of average $0.12 \pm 0.06 \times 10^{-3}$ $\mathrm{mm}^{2} / \mathrm{sec}$ (range; [0.02-0.27] $\times 10^{-3} \mathrm{~mm}^{2} / \mathrm{sec}$ ). In reticular BML (Type I), it was $0.59 \pm 0.14 \times 10^{-3} \mathrm{~mm}^{2}$ $/ \mathrm{sec}$ (range; [0.30-0.85] x10 $0^{-3} \mathrm{~mm}^{2} / \mathrm{sec}$ ). In Type II (intermediate $\mathrm{BML}$ ), it was $0.75 \pm 0.15$ ) $\times 10^{-3} \mathrm{~mm}^{2}$ $/ \mathrm{sec}$ (range; [0.40- 1.13] x $10^{-3} \mathrm{~mm}^{2} / \mathrm{sec}$ ). In compact BML (Type III), it was $1.1 \pm 0.16) \times 10^{-3} \mathrm{~mm}^{2} / \mathrm{sec}$ (range; [0.67-1.45] x $10^{-3} \mathrm{~mm}^{2} / \mathrm{sec}$ ).

\section{Group A:}

This group included 46 patients $(38.3 \%)$ out of the analyzed 120 patients (100\%). They were subdivided according to the pathological nature of the lesions into 20 patients with reactive etiology (16.7\%), 6 patients showed mechanical etiology (5\%), 18 patients with ischemic lesions (15\%), 1 patient $(0.8 \%)$ showed mechanical \& reactive insults and another 1 patient showed both ischemic and reactive etiologies as shown in table (9).

Table (9): Pathological diagnosis of lesions in group A

\begin{tabular}{|l|c|c|}
\hline \multicolumn{1}{|c|}{ Etiology } & Number & Percentage \\
\hline Reactive & 20 & $16.7 \%$ \\
\hline Mechanical & 6 & $5 \%$ \\
\hline Ischemic & 18 & $15 \%$ \\
\hline Mechanical \& Reactive & 1 & $\mathbf{0 . 8 \%}$ \\
\hline Ischemic \& Reactive & 1 & $\mathbf{0 . 8 \%}$ \\
\hline
\end{tabular}

Comparing PD and ADC values in group A revealed:

When analyzing the 46 patients of group A, in FSproton density sequence, the lesions' diameter ranged from $3.0-16.0 \mathrm{~mm}$ with a mean of $8.52 \pm 3.02 \mathrm{~mm}$, while the corresponding diameters at the ADC map ranged from 7.0 up to $36.0 \mathrm{~mm}$ with a mean of $17.85 \pm$ $5.93 \mathrm{~mm}$ (Table 10).

Table (10): Analytical description of FS-PD WI values in comparison to ADC map value among the study A group

\begin{tabular}{|c|c|c|c|c|c|}
\hline \multicolumn{2}{|c|}{$\begin{array}{c}\text { Value } \\
\text { description }\end{array}$} & $\begin{array}{c}\text { ADC map } \\
(\mathbf{m m})\end{array}$ & $\begin{array}{c}\text { FS-PD } \\
\text { WI } \\
(\mathbf{m m}) \\
\end{array}$ & $\mathbf{Z}$ & p \\
\hline \multirow{8}{*}{ 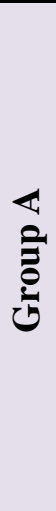 } & $\begin{array}{c}\text { Number of } \\
\text { cases }\end{array}$ & \multicolumn{2}{|c|}{46} & \multirow{7}{*}{$\frac{*}{\sigma}$} & \multirow{7}{*}{$\begin{array}{l}\text { *. } \\
\stackrel{*}{8} \\
0 \\
0\end{array}$} \\
\hline & Mean & 17.85 & 8.52 & & \\
\hline & SD & 5.93 & 3.02 & & \\
\hline & Minimum & 7 & 3 & & \\
\hline & Maximum & 36 & 16 & & \\
\hline & \multirow{2}{*}{ Median } & 17 & 8 & & \\
\hline & & $(14.5-20.0)$ & $(6.8-10.0)$ & & \\
\hline & $\begin{array}{c}\text { Ratio } \\
\text { (ADC/PD) }\end{array}$ & \multicolumn{2}{|r|}{$2.15 \pm 0.37$} & & \\
\hline
\end{tabular}

\section{Z: Wilcoxon signed ranks test}

$\mathrm{p}$ : $\mathrm{p}$ value for comparing between ADC and $\mathbf{P D}$

$*$ : Statistically significant at $\mathrm{p} \leq 0.05$

\section{Group B:}

Group B included 74 patients $(61.7 \%)$ out of the analyzed 120 patients. They were subdivided into 5 groups according to the etiology: 24 patients (20\%) showed reactive etiology, 22 patients $(18.3 \%)$ showed mechanical causes, 18 patients $(15 \%)$ represented by ischemic etiologies, 2 patients $(1.7 \%)$ showed combined mechanical and reactive cause and another 8 patients $(6.7 \%)$ showed both ischemic and reactive causes (Table 11).

Table (11): Pathological diagnosis of lesions in group B

\begin{tabular}{|l|c|c|}
\hline \multicolumn{1}{|c|}{ Etiology } & Number & Percentage \\
\hline Reactive & $\mathbf{2 4}$ & $\mathbf{2 0} \%$ \\
\hline Mechanical & 22 & $18.3 \%$ \\
\hline Ischemic & 18 & $15 \%$ \\
\hline Mechanical \& Reactive & 2 & $1.7 \%$ \\
\hline Ischemic \& Reactive & $\mathbf{8}$ & $6.7 \%$ \\
\hline
\end{tabular}




\section{Comparing PD and ADC values in group $B$ revealed:}

When analyzing the 74 patients of group B, which included patients with large BMLs showing AP diameter more than $15 \mathrm{~mm}$ in FS-PD and all of them were of type III major compact pattern of edema in ADC maps. We used volumetric assessment to minimize partial volume effects and systematic errors derived from different spacing between sections.

Their volume was measured by using the Horos "pencil" region of interest feature (ROI). Irregular regions of interest were drawn on contiguous areas of signal intensity alteration; the defined areas were then summarized and multiplied by the section thickness to calculate the BML volume by the software of Horos.

In FS-proton density sequence the lesions volume ranged from $0.90-64.0 \mathrm{~cm}^{3}$ with a mean of $11.27 \pm 12.27 \mathrm{~cm}^{3}$. While the corresponding volume at the ADC map ranged from 0.80 up to $107.0 \mathrm{~cm}^{3}$ with a mean of $21.65 \pm 20.80 \mathrm{~cm}^{3}$ (Table 12).

Table (12): Analytical description of FS-PD WI volume in comparison to ADC map volumes among the study group B

\begin{tabular}{|c|c|c|c|c|c|}
\hline & $\begin{array}{l}\text { Volume } \\
\text { values }\end{array}$ & $\begin{array}{c}\text { ADC map } \\
\left(\mathrm{cm}^{3}\right)\end{array}$ & $\begin{array}{c}\text { FS-PD WI } \\
\left(\mathrm{cm}^{3}\right)\end{array}$ & $\mathbf{Z}$ & $\mathbf{P}$ \\
\hline \multirow{7}{*}{ 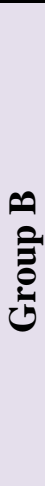 } & \begin{tabular}{|c} 
Number of \\
cases
\end{tabular} & \multicolumn{2}{|c|}{74} & \multirow{6}{*}{$\frac{*}{\stackrel{*}{*}}$} & \multirow{6}{*}{$\begin{array}{l}\stackrel{*}{8} \\
\dot{0} \\
\dot{0}\end{array}$} \\
\hline & Mean & 21.65 & 11.27 & & \\
\hline & SD & 20.80 & 12.17 & & \\
\hline & Minimum & 0.80 & 0.30 & & \\
\hline & Maximum & 107.0 & 64.0 & & \\
\hline & $\begin{array}{c}\text { Median } \\
\text { (IQR) }\end{array}$ & $\begin{array}{c}15.0 \\
(5.1-32.3) \\
\end{array}$ & $\begin{array}{c}7.0 \\
(2.5-17.3) \\
\end{array}$ & & \\
\hline & $\begin{array}{c}\text { Ratio } \\
\text { (ADC/PD) }\end{array}$ & \multicolumn{3}{|c|}{$2.12 \pm 0.40$} & \\
\hline
\end{tabular}

$\mathrm{Z}$ : Wilcoxon signed ranks test

p: $p$ value for comparing between ADC and PD

*: Statistically significant at $\mathrm{p} \leq 0.05$

\section{Concordance of BMLs:}

All the BMLs seen at the FS-PD were also detected at the ADC maps showing concordant lesions. While the ADC maps showed additional bone marrow lesions only detected at the ADC maps and could not be detected at the FS-PD. About 27 more lesions in 27 patients and 1 patient showed two more lesions. These non-concordant lesions accounted totally for 29 more lesions.

\section{DISCUSSION}

Klengel et $\boldsymbol{a l}$. (5) hypothesis reported that functional imaging with DWI would be more sensitive than conventional imaging with FS PD-weighted TSE acquisitions for the detection of BMLs. For this purpose, we compared the number and size of detected BMLs in both DWI-derived ADC maps with FS-PD weighted images for the detection of BMLs in traumatic and non-traumatic knee lesions.

There was multiple different symptoms accompanied knee bone marrow edema. The most common presented symptom was pain. Pain and click in 60 patients $(46.1 \%)$ and solitary knee pain in 30 patients $(23.05 \%)$. In addition, 20 patients showed pain and knee swelling $(15.4 \%), 7$ patients with pain, click and knee swelling $(5.4 \%)$ and 5 patients adding upon the previously mentioned symptoms limited knee movement $(3.85 \%)$. While, 4 patients presented by pain and limited movements and another 4 presented with limitation of knee (accounting for $3.1 \%$ each).

The most encountered symptom was pain either alone or accompanied by other symptoms, this is matching with Hofmann et al. ${ }^{(7)}$ who reported that the pain is caused by the increased intra-osseous pressure (normal pressure; 20-30 $\mathrm{mm} \mathrm{Hg}$ ) because of the abnormally high fluid content in the marrow spaces. In addition, we match with him at 2003 when he said that characteristic symptom of BME in the knee is pain during mechanical loading combined with other symptoms.

In our study we had no explanation why the intensity or extent of BME in MRI does not always correlate with pain. Major and Helms ${ }^{(8)}$ reported that sometimes BME of the knee is observed in asymptomatic patients. On the other hand, BME is sometimes the only definite morphologic alteration in long-lasting complaints.

In our study, we categorized the patients according to the cause of bone marrow edema into three pathological groups including; 1) Ischemic BME 2) Mechanical BME and 3) Reactive BME.

Manenti et al. ${ }^{(9)}$ conducted a study in 2015 to prompt attention focus on different advanced MR technologies such as Diffusion Weighted Imaging (DWI), Perfusion Imaging (PWI), T2 Mapping and Magnetic Resonance Spectroscopy (MRS). They mentioned that diffusion had proposed as a promising tool to assess several skeletal diseases, giving its ability to depict water shifting between different tissue compartments. Contrarily to other organs where diffusion is restricted in ischemic condition, the majority of papers showed its signal increase ${ }^{(1)}$. This phenomenon is likely due to the different cell population, which constitutes the bone marrow and to a late identification of the pathologic process, when cells' death with membranes lysis and BME has already occurred. Previous animal models, indeed, were in agreement with the theory of a transient restriction followed by an increase in diffusion of water molecules ${ }^{(10)}$. Although there is common agreement on diffusion increase in adult, analyzed 
diffusion in different disease stages, showed no significant difference.

Mueller et al. (11) reported that despite the evidence of diffusion increase in the epiphysis and metaphysis, they failed to demonstrate any difference in Apparent Diffusion Coefficient (ADC) between transient bone marrow edema, AVN, and fractures and other pathologies.

\section{Conventional MRI study:}

During our routine conventional examination of the knee for detection of bone marrow edema, we diagnosed other concomitant lesions within the related soft tissue structures. The most common involved structure was the menisci accounting for $77.7 \%$ of associated pathologies, with $60.8 \%$ of the patients showing medial meniscus injury. Aydin et al. (12) initiated a study about role of diffusion weighted in meniscal injury, five years latter Naraghi and White (13) initiated another study about meniscal and ligamental injuries and they reported that more injury of the medial meniscus than the lateral with predominance of the horizontal tear as we found in our study accounting for $10 \%$ of the patients.

Similar to Hansford and Yablon (14) we found that the combined injury of anterior cruciate ligament and medial collateral ligaments were commonly diagnosed during conventional examination of the knee, in our study both of them accounted for $33 \%$ of the patients, after the medial meniscus injury.

\section{Diffusion weighted MRI study:}

2010 was a starting era for many researches about the role of diffusion weighted MR and ADC map in musculoskeletal imaging. Khoo et al. ${ }^{(15)}$ reported that the relative value of DWI sequences in addition to or as alternatives to conventional MR sequences is equivocal for many conditions. Two years later, many other studies about DWI were done. Kizlgoz et al. ${ }^{\text {(16) }}$ studied the role of diffusion-weighted imaging and apparent diffusion coefficient mapping for meniscal tears in the knee. Subsequently, Subhawong et al. ${ }^{(17)}$, Bhojwani et al. ${ }^{(18)}$ and Dallaudière et al. ${ }^{(19)}$ published more studies for verifying the added diagnostic values of DWI and ADC maps in musculoskeletal filed. Most of them concluded DWI as a very sensitive technique that is currently used in large number of musculoskeletal conditions and is a valid technique for the staging of some solid cancers. In terms of tissue characterization, DWI should be considered as a diagnostic aid. DWI features must always be compared to the appearances of the lesion on conventional MR imaging.

Klengel et al. ${ }^{(5)}$ started a study about detection bone marrow edema after knee trauma and compared between the bone marrow edema in the ADC maps derived from diffusion weighted imaging and standard Fat-saturated Proton Density-weighted Turbo Spin Echo Sequence. In our study, we decided to widen out research base to detect the role of ADC maps derived from diffusion-weighted imaging in knee bone marrow edema in both traumatic and non-traumatic patients, to evaluate the benefit and to detect the limitations of the sequence over the routine conventional sequences. Contrary to Klengel et $\boldsymbol{a l} .{ }^{(5)}$, we tried many different b values in our study to obtain the best ADC mapderived image, but with analyzing our data, we figured out that the best $b$ value was b50,800 value as they did in their study.

\section{Classification of the BME pattern in ADC map:}

We walked through the line with Klengel et al.

(5) in calcifying the BMLs according to their visual appearance considering the same definition including type I (reticular type), which showed speckled punctuate foci of signal intensity increase on ADC maps, type II (intermediate ) with confluent patches of signal intensity increase. While, Type III (compact type) was areas of focal, homogeneous signal intensity increase, which also mostly accompanied by peripheral reticular or even intermediate signal intensity increase. Taking in consideration that some of the examined patients showed more than one pattern at the same bone affected and in different bones affected at the same patient. We almost match with their findings where the most common type was type III compact lesion, followed by the intermediate then the reticular type, which showed the least percent. We found that large compacted type III BMLs in cases with sizable traumatic injury or marked $\mathrm{OA}$ changes, reflecting high bone injury. While type I coincided with mild bone injury whether traumatic or degenerative nor ischemic injury. While type II is in between the two types.

Klengel et al. (5) performed volumetric assessment for large lesion more than $15 \mathrm{~mm}$ in their transverse diameter. While, in our study we evaluated both large BML measuring more than $15 \mathrm{~mm}$ in addition to small lesions less than $15 \mathrm{~mm}$. Therefore, we divided the studied groups into two main groups, group A for small lesions less than $15 \mathrm{~mm}$ and group $\mathrm{B}$ for large lesions measuring more than $15 \mathrm{~mm}$, which underwent further volumetric assessment. Considering the largest lesion is every patient that sometimes showed more than one lesion and even some of the examined knee showed both small and large-size lesions.

N.B; we excluded 10 patient's out of the examined 130 patients; 9 patients were excluded due to technical error within the ADC diffusion sequences as we couldn't obtain clear picture for the measurement and one patient showed restricted signal due to osteomylitis. 
Group A: This group included 46 patients (38.3\%). They were subdivided according to the underlying pathological causes into: ischemic etiologies $(15 \%)$, mechanical etiologies (5\%), reactive etiologies (16.7 $\%$ ) and combined pathologies of mechanical, reactive, ischemic and reactive etiologies ( $0.8 \%$ each). Median diameter of concordant BML at group A was approximately two times larger $(2.15 \pm 0.37)$ on ADC maps $(17 \mathrm{~mm})$ than on FS PD-weighted TSE images (8 $\mathrm{mm})(\mathrm{P}<0.001)$.

Group B: Group B included 74 patients $(61.7 \%)$ out of the analyzed 120 patients. They were subdivided into 5 groups according to the etiology, 24 patients (20\%) showed reactive etiology, 22 patients $(18.3 \%)$ showed mechanical causes and 18 patients $(15 \%)$ represented by ischemic etiologies. In addition, 2 patients $(1.7 \%)$ showed combined mechanical and reactive causes and another 8 patients $(6.7 \%)$ showed both ischemic and reactive causes. We found that the median diameter of concordant large BML lesions at group B was approximately two times larger (2.12 \pm $0.40)$ on ADC maps $\left(15 \mathrm{~cm}^{3}\right)$ than on FS PD-weighted TSE images $\left(7 \mathrm{~cm}^{3}\right)(\mathrm{P}<0.001)$. Our study match with Klengel et al. ${ }^{(5)}$ study as both of us found that the median volume of BML (whatever its size) was approximately two times larger on ADC maps than on FS PD-weighted TSE images.

The most important finding in our study was the benefits of ADC-derived map, as all the BMLs seen at the FS-PD were also detected at the ADC maps showing concordant lesions. While, the ADC maps showed additional bone marrow lesions only detected at the ADC maps and could not be detected at the FSPD about 27 more lesions in 27 patients and 1 patient showed two more lesions. These non-concordant lesions accounted totally for 29 more lesions.

The importance of this study is being having clinical implications, after knee injury, careful attention should be paid to the rehabilitation process, guiding criteria including clinical presentation, such as pain and knee function, and radiologic findings. ADC map evaluation might help to better determine when and to what degree physical activities should be recommended ${ }^{(20)}$.

\section{CONCLUSION}

- Bone marrow edema (BME), with its typical signal appearance on MRI, is a common but nonspecific signal pattern that can be found during routine knee examination.

- Magnetic resonance imaging (MRI) is the mainstay of diagnosis, staging and management plan of knee traumatic and non-traumatic disorders.

- ADC maps are more sensitive than corresponding FS PD weighted TSE images for detection of BML allowing detection of significantly more and larger BMLs. In addition, they represent a valuable add-on in knee imaging protocols.

- New MRI techniques for the knee such as the ADC maps and other techniques are fast and applicable with routine MRI sequences. They can depict very important information about menisci, cartilages, bone and soft tissue of the knee as well as post-operative evaluation. Moreover, the ADC-derived map may be more useful than morphological MRI alone in knee assessment.

-

\section{REFERENCES}

1. Hong N, Du X, Nie Z, Li S (2005): Diffusion-weighted MR study of femoral head avascular necrosis in severe acute respiratory syndrome patients. J Magn Reson Imaging, 22 (5): 661-4.

2. Papadopoulos EC, Papagelopoulos PJ, Boscainos PJ et al. (2001): Bone marrow edema syndrome. Orthopedics, 24: 69-73; quiz 74-5.

3. Vogler JB, Murphy WA (2001): Diffuse marrow diseases. In: Berquest TH, editor. MRI of the musculoskeletal system. Philadelphia: Lippincott, Williams \& Wilkins, Pp: 979-1028.

4. Mayerhoefer ME, Breitenseher MJ, Kramer J et al. (2005): STIR vs. T1-weighted fat suppressed gadolinium-enhanced MRI of bone marrow edema of the knee: computer-assisted quantitative comparison and influence of injected contrast media volume and acquisition parameters. J Magn Reson Imag., 22: 788-93.

5. Klengel A, Stumpp P, Klengel $S$ et al. (2016): Detection of Traumatic Bone Marrow Lesions after Knee Trauma: Comparison of ADC Maps Derived from Diffusion-weighted Imaging with Standard Fat-saturated Proton Density-weighted Turbo Spin-Echo Sequences. Radiology, 8: 1-9.

6. Ward R, Caruthers S, Yablon C et al. (2000): Analysis of diffusion changes in posttraumatic bone marrow using navigator-corrected diffusion gradients. AJR Am J Roentgenol., 174 (3): 731-4.

7. Hofmann S, Kramer J, Breitenseher M et al. (2003): The painful bone marrow edema of the knee [Das schmerzhafte Knochenmarko"dem im Kniegelenk]. Arthroskopie, 16 (2): $88-100$.

8. Major NM, Helms CA (2002): MR imaging of the knee: findings in asymptomatic collegiate basketball players. AJR Am J Roentgenol., 179 (3): 641-4.

9. Manenti G, Altobelli S, Pugliese L et al. (2015): The role of imaging in diagnosis and management of femoral head avascular necrosis. Clinical Cases in Mineral and Bone Metabolism, 12 (1): 31-8.

10. Menezes NM, Connolly SA, Shapiro F et al. (2007): Early ischemia in growing piglet skeleton: MR diffusion and perfusion imaging. Radiology, 242 (1): 129-36.

11. Mueller D, Schaeffeler C, Baum T et al. (2014): Magnetic resonance perfusion and diffusion imaging characteristics of transient bone marrow edema, avascular necrosis and subchondral insufficiency fractures of the proximal femur. Eur J Radiol., 83 (10): 1862-9. 
12. Aydin H, Kızılgoz V, Hekimoğlu B (2011): Is the quantitative Diffusion-Weighted MR Imaging and ADC mapping with $\mathrm{b}$ values of 50,400 , and $800 \mathrm{sec} / \mathrm{mm} 2 \mathrm{a}$ reliable method for evaluation of meniscal tears in the knee? Pol J Radiol., 76 (1): 30-40.

13. Naraghi AM, White LM (2016): Imaging of Athletic Injuries of Knee Ligaments and Menisci: Sports Imaging Series. Radiology, 281 (1): 23-40.

14. Hansford BG, Yablon CM (2017): Multiligamentous Injury of the Knee: MRI Diagnosis and Injury Patterns. Semin Musculoskelet Radiol., 21: 63-74.

15. Khoo MMY, Tyler PA, Saifuddin A et al. (2011): Diffusion-weighted imaging (DWI) in musculoskeletal MRI: a critical review. Skeletal Radiol., 40: 665-81.

16. Kızılgoz V, Aydın H, Hekimoğlu B (2013): The efficacy of diffusion weighted imaging and apparentdiffusion coefficient mapping for meniscal tears in the knee. Science Journal of Clinical Medicine, 2 (6): 171-5.
17. Subhawong TK, Jacobs MA, Fayad LM (2014): Diffusion-weighted MR Imaging for characterizing Musculoskeleta lesions. Radio Graphics, 34:1163-77.

18. Bhojwani N, Szpakowski P, Partovi S et al. (2015): Diffusion-weighted imaging in musculoskeletal radiology — clinical applications and future directions. Quant Imaging Med Surg., 5 (5): 740-53.

19. Dallaudière $B$, Lecouvet $F$, Vande Berg $P$ et al. (2015): Diffusion-weighted MR imaging in musculoskeletal diseases: Current concepts. Diagnostic and Interventional Imaging, 96: 327-40.

20. Van Ginckel A, Verdonk P, Witvrouw E (2013): Cartilage adaptation after anterior cruciate ligament injury and reconstruction: implications for clinical management and research? A systematic review of longitudinal MRI studies. Osteoarthritis Cartilage, 21 (8): 1009-24. 